\title{
Assessment of cardiac function in donor and recipient fetuses during a 7-day follow-up after selective laser photocoagulation of communicating vessels due to TTTS
}

\author{
Katarzyna Leszczynska ${ }^{1}$, Jaroslaw Meyer-Szary ${ }^{2}$, Maciej Chojnicki ${ }^{3}$, \\ Ireneusz Haponiuk ${ }^{3,4}$, Krzysztof Preis ${ }^{1}$, Katarzyna Stefanska ${ }^{1}$, \\ Katarzyna Gierat-Haponiuk ${ }^{5}$, Malgorzata Swiatkowska-Freund ${ }^{1}$ \\ ${ }^{1}$ Medical University of Gdansk, Department of Obstetrics, Gdansk, Poland \\ ${ }^{2}$ Medical University of Gdansk, Department of Pediatric Cardiology and Congenital Heart Diseases, Gdansk, Poland \\ ${ }^{3}$ Copernicus Hospital in Gdansk, Department of Pediatric Cardiac Surgery, Gdansk, Poland \\ ${ }^{4}$ Gdansk University of Physical Education and Sport, Chair of Physiotherapy, Gdansk, Poland \\ ${ }^{5}$ Medical University of Gdansk, Department of Rehabilitation, Gdansk, Poland
}

\begin{abstract}
Objectives: The aim of the study was to analyze the changes in cardiac function and myocardial contractility of donor and recipient fetuses with twin-to-twin transfusion syndrome (TTTS) subjected to selective laser photocoagulation of the communicating vessels (SLPCV), between and after the procedure. Finally, we verified if fetuses with Quintero's stage ITTTS presented with early impairment of myocardial contractility.

Material and methods: We selected 77 consecutive women with twin pregnancies, whose both fetuses survived at least seven days post-SLPCV. Myocardial contractility of both fetuses was evaluated ultrasonographically, and their myocardial performance indices (Tei-Index values) and shortening fractions (SF) were determined.

Results: In donor fetuses, the Tei-Index values for both right and left ventricle remained within the respective reference ranges both before the procedure and during a 7-day follow-up. A significant change in shortening fraction values for the left ventricle in recipient fetuses and the right ventricle of in the donors was observed during a 7-day follow-up.

Conclusions: Comparison of the cardiac parameters of donors and recipients revealed significant differences in Tei-indices during the entire follow-up period.

The group with Quintero's I stage TTTS included 74\% of recipient fetuses with abnormal Tei-Index values for the right ventricle (mean 0.53).

Key words: SLPCV; donor; recipient; heart assessment; laser photocoagulation; Tei-Index
\end{abstract}

Ginekologia Polska 2019; 90, 4: 189-194

\section{INTRODUCTION}

Twin-to-twin transfusion syndrome (TTTS) is a severe pathology of multifetal monochorionic pregnancy. TTTS results from imbalanced blood flow in placental communicating vessels, which leads to the development of hypovolemia in one fetus, referred to as the donor, and hypervolemia in another fetus referred to as the recipient. Untreated TTTS results in the death of both fetuses in $80-100 \%$ of the cases [1,2]. TTTS is diagnosed during pregnancy, on the basis of ultrasonographic examination.

The most widespread scale used for classification of the severity of TTTS is the staging system proposed by Ruben Quintero in 1999 (Tab. 1) [3].

However, the abovementioned scoring system does not include parameters describing the myocardial function of both fetuses. A few scales for echocardiographic assessment 
Table.1. TTTS severity scale by Ruben Quintero

\begin{tabular}{|l|l|l|l|l|l|}
\hline Symptom/stage & I & II & III & IV & V \\
\hline Polyhydramnios/oligohydramnios & + & + & + & + & + \\
\hline Absent bladder & - & + & + & + & + \\
\hline Abnormal Doppler flows (UA, UV, DV, TV) & - & - & + & + & + \\
\hline Generalized hydrops & - & - & - & + & + \\
\hline Demise of at least one fetus & - & - & - & - & + \\
\hline
\end{tabular}

DV — venous duct; TV — tricuspid valve; UA — umbilical artery; UV - umbilical vein

of twin pregnancies have been developed thus far, among them the Children's Hospital of Philadelphia (CHOP) Scoring System proposed by Rychik in 2007.

In the CHOP system, each parameter is scored according to its presence and severity, with the maximum score of 20 . Based on the final score, the severity of TTTS is graded from I (mild) to IV (most severe). To carry out such a detailed assessment, the examiner needs to have adequate experience in echocardiography and a sufficient amount of time [4, 5].

Another scoring system is Cardiovascular Profile Score by Huhta (2004), which was first used to in order to evaluate fetuses from multiple pregnancies by Shah in 2008. Aside from Doppler evaluation of blood flow in the umbilical vessels and examination for potential fetal hydrops, also the presence of cardiomegaly and myocardial performance are considered during the determination of the Cardiovascular Profile Score [6].

The Cincinnati Scale proposed by Habli et al. [7] in 2008 is based on the presence and degree of atrioventricular valve insufficiency, myocardial thickness and ventricular function expressed by myocardial performance index (MPI, Tei-Index).

Finally, Stirnemann Scale was proposed in 2010. According to this scale, the most sensitive marker of fetal heart function is the myocardial performance index, Tei-Index [8].

Currently, the treatment of choice in TTTS entails causal management, namely selective laser photocoagulation of the communicating vessels (SLPCV) [9]. This method involves insertion of a trocar with fetoscope to the amniotic cavity (usually to the recipient's amniotic sac) and identification of communicating vessels on the placental surface. Subsequently, an optical fiber is inserted through another work channel of the trocar to photocoagulate selected blood vessels with an infrared laser beam (Fig. 1, 2).

The aim of the SLPCV is to separate fetal circulations, and thus, to change the monochorionic pregnancy into a hemodynamically dichorionic pregnancy. Photocoagulation causes immediate closure of the communicating vessels, which contributes to a sudden change of hemodynamic conditions. Response to this change is determined by the myocardial status of the fetal heart before the procedure and

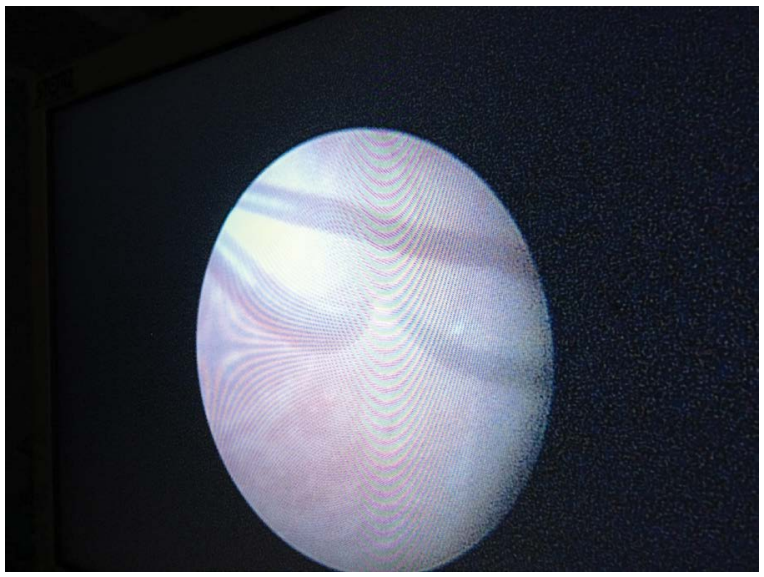

Figure 1. Fetoscopic image: placental vessels

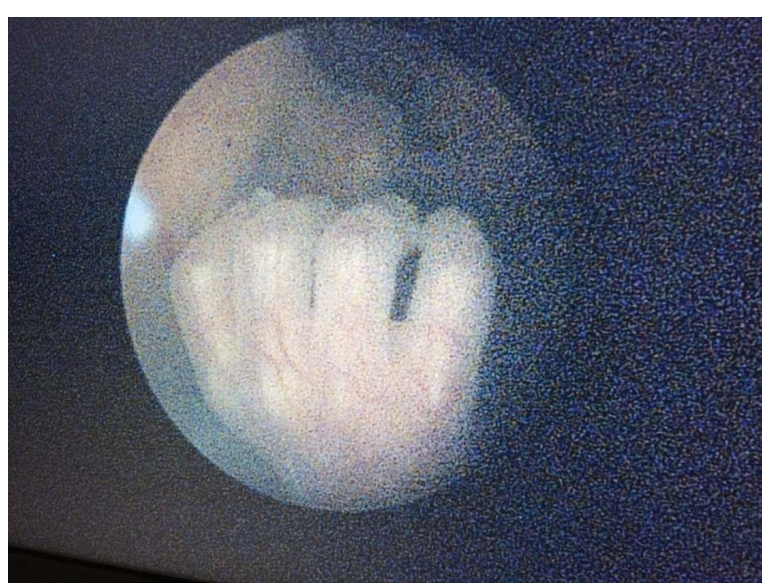

Figure 2. Fetoscopic image: the hand of the fetus

its adaptive potential [2]. Echocardiographic assessment of fetal heart before the SLPCV seems to be a prognostic factor for further outcome in twin pregnancy $[10,11]$.

\section{Objectives}

The aim of the study was to analyze the changes in cardiac function and myocardial contractility of donor and recipient fetuses with TTTS subjected to SLPCV, between the first day prior to and the seventh day after the procedure. We also compared the parameters of cardiac function in donor and recipient fetuses before and after the SLPCV. Finally, we verified if fetuses with Quintero's stage ITTTS presented with early impairment of myocardial contractility.

\section{MATERIAL AND METHODS}

From a group of 142 women subjected to SLPCV due to TTTS, 77 consecutive patients with twin pregnancies, in whom this procedure was carried out at the Department of Obstetrics, Medical University of Gdansk, between January 1st, 2009 and August 1st, 2015, and whose both fetuses 


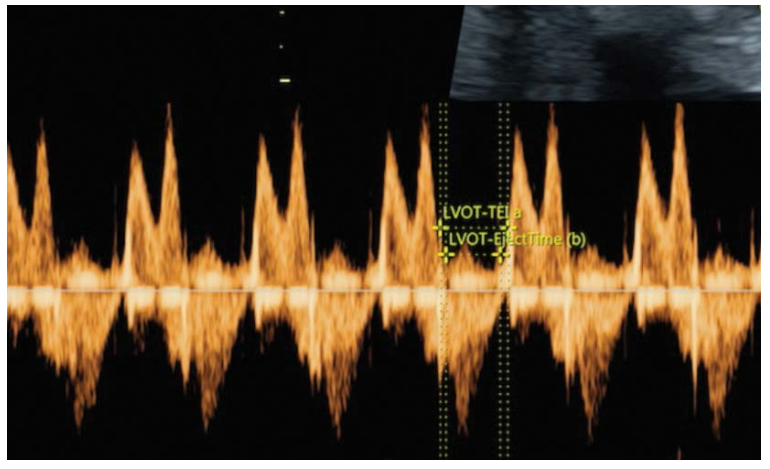

Figure 3. Tei-Index measurement

survived at least seven days post-procedure were selected. Fetal ultrasonographic examination was conducted one day before the SLPCV, as well as 1, 3(4) and 7 days thereafter. The study patients were qualified to SLPCV based on their Quintero's scores [3, 12, 13].

Myocardial contractility of both fetuses was evaluated ultrasonographically, and their myocardial performance indices (Tei-Index values) and shortening fractions (SF) were determined. We used the reference values for Tei-Index proposed by Falkensammer and Huhta, i.e. $0.38 \pm 0.05$ and $0.41 \pm 0.05$ for the right and left ventricle Tei-Index, respectively. SF was considered normal if it amounted to $25-45 \%$; SF between $15 \%$ and $25 \%$ was interpreted as a mild decrease, and SF $<15 \%$ as severe cardiac dysfunction [14].

Each measurement was taken in triplicate, once during each of three consecutive cardiac cycles, and arithmetic mean from the three measurements was subjected to further analysis (Fig. 3).

\section{Inclusion criteria}

The study included women with twin pregnancies complicated with TTTS, who required SLPCV between 16 and 26 weeks of gestation. Only the patients whose both fetuses survived at least seven days post-procedure were subjected to the analysis. SLPCV was performed by two operators (MSF and KP). Pre- and post-procedural ultrasonographic scans were obtained by two ultrasonographers (MSF and KL), using a standardized protocol of examination.

\section{Exclusion criteria}

Two groups of patients were excluded from the hereby presented analysis and their results will be presented elsewhere.

The first group included women with twin pregnancies, in whom digitalis preparations needed to be implemented before the SLPCV due to severe cardiac dysfunction in recipient fetus (SF $<15 \%$ ). As distinguishing between the effects of SLPCV and previous pharmacotherapy on myocar- dial contractility was challenging, we excluded these cases from the analysis, and their outcomes will be a subject of a separate study. This preliminary observation highlights the importance of correct early diagnosis and early implementation of SLPCV in fetuses (especially recipients) with a good myocardial condition.

The second group of patients excluded from the study were the women in whom digitalis preparations were administered after the SLPCV due to the evidence of hydrops in donor fetus. It needs to be emphasized that we refrained from the administration of digitalis whenever transient tricuspid regurgitation was detected in donor fetus.

Statistical analysis was carried out with Wizard Pro 1.9.13 software (Evan Miller Chicago, IL). Categorical variables were expressed as numbers ( $n$ ) and percentages, and continuous variables as means \pm standard deviations or medians (minimum-maximum), depending on their distribution. Normality of the distribution was tested with the Shapiro-Wilk test. Paired Student t-test and Wilcoxon signed-rank test for repeated measures were used for intragroup comparisons, whereas the intergroup comparisons were carried out with Student t-test and Mann-Whitney U-test. Multiple comparisons were conducted with parametric ANOVA or Friedman test. Distributions of categorical variables were compared with the chi-square test, McNemar test and Stuart-Maxwell test if the changes over time were analyzed. The threshold of statistical significance was set at $p<0.05$.

\section{RESULTS}

The parameters specified in the study protocol were determined in the group of 77 patients, before SLPCV and during a 7-day follow-up after the procedure. Complete results and the outcomes of statistical analysis are presented in Table 2.

Myocardial performance indices and shortening fraction values for both ventricles in donor and recipient fetuses, determined before SLPCV, as well as 1, 3(4) and 7 days postprocedure, are presented in Table 2 . In donor fetuses, the TeiIndex values for both right and left ventricle remained within the respective reference ranges both before the procedure and during a 7-day follow-up. In recipient fetuses, the values of myocardial performance indices were abnormal before SLPCV, improved slightly after the successful procedure (with success defined as resolution of TTTS symptoms), but remained beyond the respective reference ranges on day 7 .

A significant change in shortening fraction values for the left ventricle in recipient fetuses and the right ventricle in the donors was observed during a 7-day follow-up, as shown in Figure 4.

Comparison of the cardiac parameters of donors and recipients revealed significant differences in Tei-indices during the entire follow-up period. Moreover, a significant inter- 
Table 2. Changes in Tei-Index and SF values for recipient and donor fetuses, observed during a 7-day follow-up after SLPCV. The inner cells provide descriptive statistics of the measurements, the outer cells provide $p$ values for changes in time (right-hand column), and for comparison of corresponding measures for each pair of donor and recipient (below each pair). Statistically significant differences marked bold

\begin{tabular}{|c|c|c|c|c|c|c|c|}
\hline \multirow{2}{*}{$\begin{array}{l}\text { Para- } \\
\text { meter }\end{array}$} & \multirow{2}{*}{ Ventricle } & \multirow{2}{*}{ Fetus } & \multicolumn{4}{|l|}{ Day of follow-up } & \multirow{2}{*}{$\begin{array}{l}\text { Change in } \\
\text { time }\end{array}$} \\
\hline & & & 1 day before & 1 day after & 3(4) days after & 7 days after & \\
\hline \multirow{6}{*}{$\begin{array}{l}\text { Tei } \\
\text { Index }\end{array}$} & \multirow{3}{*}{ LV } & Donor & $0.38(0.30-0.63)$ & $0.35(0.28-0.68)$ & $0.37(0.26-0.57)$ & $0.38(0.26-0.71)$ & $p=0.960$ \\
\hline & & Recipient & $0.49(0.29-0.85)$ & $0.46(0.24-1.10)$ & $0.45(0.37-0.80)$ & $0.46(0.32-0.80)$ & $p=0.833$ \\
\hline & & Don. vs Rec. & $\mathrm{p}<0.001$ & $p<0.001$ & $p<0.001$ & $p<0.001$ & \\
\hline & \multirow{3}{*}{ RV } & Donor & $0.35(0.20-0.66)$ & $0.35(0.25-0.74)$ & $0.37(0.23-0.66)$ & $0.38(0.25-0.61)$ & $p=0.108$ \\
\hline & & Recipient & $0.47(0.25-1.05)$ & $0.40(0.25-1.50)$ & $0.46(1.25-1.09)$ & $0.47(0.25-1.09)$ & $p=0.659$ \\
\hline & & Don. vs Rec. & $p<0.001$ & $p<0.001$ & $p<0.001$ & $p=0.005$ & \\
\hline \multirow{6}{*}{ SF } & \multirow{3}{*}{ LV } & Donor & $46.3 \pm 5.7$ & $39.7 \pm 8.2$ & $41.3 \pm 6.8$ & $45.5 \pm 5.3$ & $p=0.463$ \\
\hline & & Recipient & $41.30 \pm 7.0$ & $45.1 \pm 15.1$ & $47.3 \pm 11.9$ & $43.4 \pm 11.7$ & $p=0.026$ \\
\hline & & Don. vs Rec. & $p=0.022$ & $p=0.198$ & $p=0.038$ & $p=0.507$ & \\
\hline & \multirow{3}{*}{ RV } & Donor & $40.8 \pm 3.8$ & $37.7 \pm 8.6$ & $42.5 \pm 8.7$ & $43.4 \pm 6.5$ & $p=0.006$ \\
\hline & & Recipient & $37.5 \pm 16.3$ & $32.0 \pm 16.9$ & $33.2 \pm 14.8$ & $35.2 \pm 14.9$ & $p=0.339$ \\
\hline & & Don. vs Rec. & $p=0.240$ & $p=0.240$ & $p=0.011$ & $p=0.051$ & \\
\hline
\end{tabular}

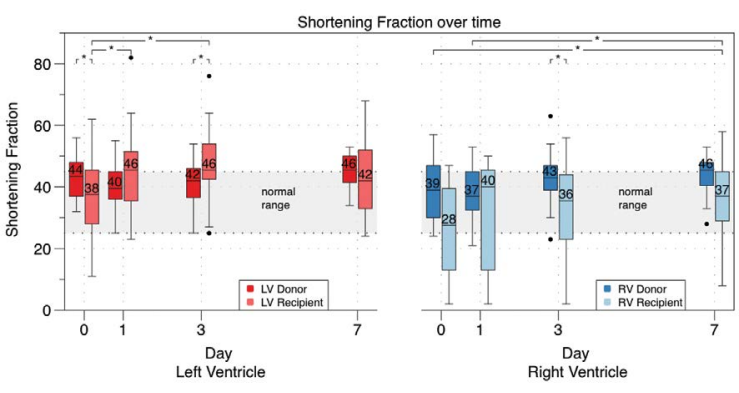

Figure 4. Changes in shortening fractions for donor and recipient fetuses determined before the SLPCV and during a 7-day postprocedure follow-up, and comparisons of the shortening fractions for donors and recipients

group difference in the shortening fraction for the left ventricle was found one day before SLPCV and 3(4) post-SLPCV, along with a difference in the shortening fraction for the right ventricle on days 3 and 7 (for the latter time point the difference was at a threshold of statistical significance) (Fig. 5).

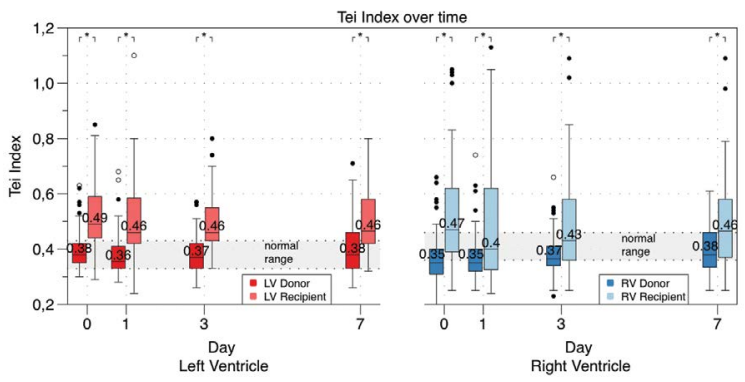

Figure 5. Changes in Tei-Indices for donor and recipient fetuses determined before the SLPCV and during a 7-day post-procedure follow-up, and comparison of the Tei-indices in donors and recipients

\begin{tabular}{|l|l|l|l|}
\multicolumn{4}{|c|}{ Table 3. Measurements of Tei-Index in Quintero's first stage } \\
\hline Column & Count & Mean (range) & $\%$ of abnormal TEI \\
\hline TEI LV D & 19 & $0.35(0.29-0.52)$ & $6 \%$ \\
\hline TEI RV D & 19 & $0.35(0.24-0.46)$ & $11 \%$ \\
\hline TEI LV R & 19 & $0.44(0.34-0.8)$ & $37 \%$ \\
\hline TEI RV R & 19 & $0.53(0.36-1.94)$ & $74 \%$ \\
\hline
\end{tabular}

To satisfy the third objective of the study, a subset of 19 (25\%) women with Quintero's stage I TTTS was identified among the 68 patients whose both fetuses survived at least seven days post-procedure. The group with Quintero's I stage TTTS included $74 \%$ of recipient fetuses with abnormal Tei-Index values for the right ventricle (mean 0.53) and borderline Tei-Index values for the left ventricle (mean 0.44). Mean Tei-Index values for the left and right ventricle of donor fetuses were within the respective reference ranges and amounted to 0.35 and 0.35 , respectively (Tab. 3).

These findings imply that most fetuses with TTTS present with anomalies of cardiac function already before the manifestation of other pathological features included in the most widespread Quintero's scale, and the right ventricle in the recipients is affected more than the left ventricle.

\section{DISCUSSION}

SLPCV is the most efficient and effective of currently available management options in TTTS. However, it is still unclear if all fetuses subjected to SLPCV can fully adjust to the new hemodynamic conditions, namely increased blood volume in donors and reduced blood volume in recipients.

Fetuses presenting with impaired myocardial function before SLPCV have a markedly worse prognosis than the 
other patients. Thus, improvement of TTTS detection rate is of vital importance, as it will enable to implement SLPCV at earlier clinical stages.

Previous studies showed that the post-SLPCV decrease in recipient's blood volume might often improve both right and left ventricular function, which manifests as an increase in myocardial contractility $[15,16]$.

Our findings are slightly different from those reported previously. Habli et al. [10] documented a beneficial effect of improved myocardial contractility in recipient fetuses on pregnancy outcome and found a positive correlation between the post-SLPCV increase in recipient's contractility and the survival after the procedure.

Papanna et al. [11] determined myocardial contractility in recipients 24 hours before SLPCV, during the procedure, and 12 hours and 24 hours thereafter; the increase in myocardial contractility was observed both during and after the procedure.

Stirnemann et al. [17] did not find a significant association between the post-SLPCV decrease in myocardial contractility in donor fetuses (defined as an increase in Tei-Index or a decrease in SF) and worse outcome of pregnancies in which laser photocoagulation was implemented as the first-line treatment. In our present study, SLPCV contributed to a significant decrease in SF values, for the left ventricle in recipients and the right ventricle in donors. However, we did not find statistically significant post-procedural changes in Tei-indices in either recipients or donors. Our findings highlight another practical aspect of myocardial contractility assessment: analysis of this parameter as early as on the first day post-SLPCV can confirm that most, if not all, communicating vessels were efficiently closed during the procedure. Not infrequently, donor's amniotic fluid and/or urinary bladder still cannot be visualized on the first day after SLPCV. However, either a trace of tricuspid regurgitation or a slight decrease in myocardial contractility can be already observed in donor fetuses on the first day, pointing to enhanced cardiovascular strain and being, in fact, a favorable prognostic factor.

As expected, cardiac parameters of donor and recipient fetuses differed significantly, both before the SLPCV and during a 7-day post-procedural follow-up.

Future studies with longer follow-up should explain how many days are needed for complete normalization of myocardial function in recipient fetuses after SLPCV.

We still analyze the data for the two groups of patients excluded from our present study due to the administration of digitalis preparations. In the first group, digitalis was given before the SLPCV because of poor myocardial performance in recipient fetuses, and in the second postoperatively, due to the evidence of donor hydrops. These groups are going to be a subject of separate comprehensive research the results of which will be presented in future.

In our opinion, the most interesting finding of this study is the observation that nearly $74 \%$ of recipient fetuses subjected to SLPCV due to Quintero's stage I TTTS presented with abnormal right ventricular myocardial function, and $37 \%$ of the recipients had both left and right ventricular dysfunction. This implies that impairment of myocardial contractility precedes manifestation of other characteristic features of TTTS included in the Quintero's scale. Perhaps, more careful observation and more accurate measurement of myocardial contractility indices, beginning already in the 1st trimester, would facilitate earlier identification of pregnancies at risk of TTTS, before the changes in amniotic fluid volume emerged [18]. Identification of cut-off values forTei-Index distinguishing accurately between the high-risk and low-risk group would enable physicians to focus more on the former group and to offer them more frequent control visits (for example, once a week rather than every second week). As a result, most cases of TTTS could be identified and treated by SLPCV already at Quintero's stage I. It is still unclear whether the laser treatment in Quintero stage I is necessary, because only $27 \%$ of TTTS cases progress to higher stages [19]. Perhaps, the early echocardiographic examination could identify the group of fetuses with worse cardiac function (i.e. the $37 \%$ of fetuses with Quintero's stage ITTTS and both left and right ventricular dysfunction) and it would be the same group with worse prognosis which needs SLPCV.

The changes in myocardial contractility of fetuses subjected to SLPCV are a subject of ongoing research. The fact that a growing number of patients are subjected to this procedure raises a possibility to understand better all pathogenic mechanisms of TTTS, and perhaps also to develop the algorithms for pre- and post-SLPCV management, adjusted for fetal echocardiographic findings. Definitively, studies with long-term follow-up are needed [20]. Accurate prenatal diagnostics, including a detailed evaluation of fetal heart and its potential pathologies, with no doubt, contributes to an improvement of neonatal safety and better quality of multimodal perinatal care [21, 22].

\section{CONCLUSIONS}

SLPCV improves myocardial contractility in recipient fetuses with TTTS.

Improvement of myocardial contractility in twin fetuses might be an early prognostic factor for the positive outcome of SLPCV.

In most cases of TTTS (74\%), a decrease in myocardial contractility of recipient fetuses was observed already at Quintero's stage I. 


\section{REFERENCES}

1. Victor YH. Risk in multiple births especially from twin-to-twin transfusion syndrome. Circulation. 2002; 106: 585-591.

2. Herberg U, Gross W, Bartmann P, et al. Long term cardiac follow up of severe twin to twin transfusion syndrome after intrauterine laser coagulation. Heart. 2006; 92(1): 95-100, doi: 10.1136/hrt.2004.057497, indexed in Pubmed: 15814592.

3. Quintero RA, Dickinson JE, Morales WJ, et al. Stage-based treatment of twin-twin transfusion syndrome. Am J Obstet Gynecol. 2003; 188(5): 1333-1340, indexed in Pubmed: 12748508.

4. Diagnosis and Staging of Twin to Twin Transfusion Syndrome. www. perinatology.com.

5. Davey BT, Donofrio MT, Moon-Grady AJ, et al. Development and validation of a fetal cardiovascular disease severity scale. Pediatr Cardiol. 2014; 35(7): 1174-1180, doi: 10.1007/s00246-014-0911-9, indexed in Pubmed: 24801674.

6. Shah AD, Border WL, Crombleholme TM, et al. Initial fetal cardiovascular profile score predicts recipient twin outcome in twin-twin transfusion syndrome. J Am Soc Echocardiogr. 2008; 21(10): 1105-1108, doi: 10.1016/j.echo.2008.05.004, indexed in Pubmed: 18558475.

7. Habli M, Michelfelder E, Cnota J, et al. Prevalence and progression of recipient-twin cardiomyopathy in early-stage twin-twin transfusion syndrome. Ultrasound Obstet Gynecol. 2012; 39(1): 63-68, doi: 10.1002/uog.10117, indexed in Pubmed: 21998013.

8. Stirnemann JJ, Nasr B, Proulx F, et al. Evaluation of the CHOP cardiovascular score as a prognostic predictor of outcome in twin-twin transfusion syndrome after laser coagulation of placental vessels in a prospective cohort. Ultrasound Obstet Gynecol. 2010; 36(1): 52-57, doi: 10.1002/uog.7713, indexed in Pubmed: 20582931.

9. Quintero RA, Ishii $\mathrm{K}, \mathrm{Chmait} \mathrm{RH}$, et al. Sequential selective laser photocoagulation of communicating vessels in twin-twin transfusion syndrome. J Matern Fetal Neonatal Med. 2007; 20(10): 763-768, doi: 10.1080/14767050701591827, indexed in Pubmed: 17763279.

10. Habli M, Michelfelder E, Livingston J, et al. Acute effects of selective fetoscopic laser photocoagulation on recipient cardiac function in twin-twin transfusion syndrome. Am J Obstet Gynecol. 2008; 199(4): 412.e1-412. e6, doi: 10.1016/j.ajog.2008.06.067, indexed in Pubmed: 18722576.

11. Papanna R, Mann LK, Molina S, et al. Changes in the recipient fetal Tei index in the peri-operative period after laser photocoagulation of placental anastomoses for twin-twin transfusion syndrome. Prenat Diagn. 2011; 31(2): 176-180, doi: 10.1002/pd.2673, indexed in Pubmed: 21268037.

12. Quintero R. (ed). Twin-twin transfusion syndrome. Informa Health, London 2007.

13. Respondek-Liberska M, Janiak K. Protokół badania kardiologicznego u płodu w ośrodku referencyjnym. Pol Przegl Kardiolo. 2010;12(3):212-218.

14. Falkensammer CB, Paul J, Huhta JC. Fetal congestive heart failure: correlation of Tei-index and Cardiovascular-score. J Perinat Med. 2001; 29(5): 390-398, doi: 10.1515/JPM.2001.055, indexed in Pubmed: 11723840.

15. Preis K, Leszczyńska K, Świątkowska-Freund M, et al. Kurczliwość komór serca płodów po zabiegu laserowej okluzji naczyń łączących w TTTS. Perinatologia, Neonatologia i Ginekologia. 2009; 2(1): 48-49.

16. Leszczyńska K, Preis K, Swiatkowska-Freund M, et al. Comparison of fetal myocardial contractility before and after laser photocoagulation of communicating vessels in twin-to-twin transfusion syndrome. Ginekol Pol. 2014; 85(4): 283-286, indexed in Pubmed: 24834706.

17. Stirnemann JJ, Mougeot M, Proulx F, et al. Profiling fetal cardiac function in twin-twin transfusion syndrome. Ultrasound Obstet Gynecol. 2010; 35(1): 19-27, doi: 10.1002/uog.7488, indexed in Pubmed: 20020467.

18. Leszczyńska K, Preis K, Respondek-Liberska M, et al. Recommendations for Fetal Echocardiography in Twin Pregnancy in 2016. Prenatal Cardiology. 2016; 6(1): 6-15, doi: 10.1515/pcard-2016-0001.

19. Washburn EE, Sparks TN, Gosnell KA, et al. Stage ITwin-Twin Transfusion Syndrome: Outcomes of Expectant Management and Prognostic Features. Am J Perinatol. 2018; 35(14): 1352-1357, doi: 10.1055/s-00381627095, indexed in Pubmed: 29528469.

20. Perry H, Duffy JMN, Umadia O, et al. International Collaboration to Harmonise Outcomes for Twin-Twin Transfusion Syndrome (CHOOSE). Outcome reporting across randomized trials and observational studies evaluating treatments for twin-twin transfusion syndrome: systematic review. Ultrasound Obstet Gynecol. 2018; 52(5): 577-585, doi: 10.1002/uog.19068, indexed in Pubmed: 29607558.

21. Haponiuk I, Chojnicki M, Szofer-Sendrowska A, et al. „Planned” permanent pacemaker implantation in one-day-old newborn after prenatal diagnosis of congenital complete atrioventricular heart block. Kardiochir Torakochirurgia Pol. 2014; 11(1): 76-78, doi: 10.5114/kitp.2014.41937, indexed in Pubmed: 26336400.

22. Kornacka $M$, Respondek-Liberska $M$. The place for neonatologist in proper perinatal care. Prenatal Cardiology. 2013; 3(3): 12-15. 